\title{
A deterministic multi-tier model of assortative mating following selection
}

\author{
RK Shepherd ${ }^{1}$, BP Kinghorn ${ }^{2}$ \\ 1 Department of Mathematics and Computing, University of Central Queensland, \\ Rockhampton, Queensland 4702; \\ 2 Department of Animal Science, University of New England, Armidale, \\ New South Wales 2351, Australia
}

(Received 13 January 1994; accepted 3 June 1994)

\begin{abstract}
Summary - A deterministic model is presented of assortative mating following selection on either phenotype or best linear unbiased prediction (BLUP) estimates of breeding values (ebv) in an infinite population. The model is based on modified theory for multitier open nucleus breeding schemes. It is shown that the percentage increase in genetic gain of assortative mating over random mating is greatly increased at low to moderate heritability when BLUP rather than mass selection is used. The percentage increase in genetic gain at equilibrium of assortative mating over random mating is independent of initial heritability and family structure when selection is on BLUP ebv. The same is true in the early generations if there is ample pedigree history available before selection commences. The deterministic prediction of the percentage increase in genetic gain at equilibrium of assortative mating over random mating is 11,24 and $66 \%$ when 10,50 and $90 \%$ of progeny are selected on BLUP ebv. Stochastic simulation is used to evaluate the accuracy of the deterministic model. Both deterministic and stochastic results for assortative mating indicate a considerably increased value over random mating in certain situations than has previously been reported.
\end{abstract}

assortative mating / selection / BLUP / genetic group / open nucleus

Résumé - Un modèle déterministe d'homogamie après sélection dans un schéma à plusieurs étages. Cet article décrit un modèle déterministe pour une population infinie soumise à homogamie après une sélection soit sur le phénotype soit sur la valeur génétique estimée (vge) par le BLUP. Le modèle est basé sur la théorie modifiée des schémas de sélection à noyau ouvert à plusieurs étages. On montre que l'accroissement du gain génétique dî̀ à l'homogamie par rapport à la panmixie est grandement augmenté pour des héritabilités faibles à modérées quand on utilise le BLUP au lieu de la sélection massale. Le pourcentage d'augmentation du gain à l'équilibre quand on utilise l'homogamie de préférence à la panmixie est indépendant de l'héritabilité initiale et de la structure familiale quand la sélection se fait sur la vge BLUP. Cela est vrai aussi dans les premières générations, si les pedigrees antérieurs à la période de sélection sont bien connus. La prédiction déterministe de l'augmentation du gain génétique à l'équilibre avec l'homogamie 
par rapport à la panmixie est de $11 \%, 24 \%$ et $66 \%$, pour des taux respectifs de sélection sur la vge BLUP de 10\%, 50\% et 90\%. Une simulation stochastique a été faite pour évaluer la précision du modèle déterministe. Les résultats, aussi bien déterministes que stochastiques, montrent un avantage de l'homogamie sur la panmixie qui est, dans certaines situations, nettement supérieur aux résultats antérieurement publiés.

homogamie / sélection / BLUP / groupes génétiques / noyau ouvert

\section{INTRODUCTION}

For random mating, deterministic methods are available to predict the asymptotic response to selection for an infinite population in which the selected character is controlled by many unlinked genetic loci, each of small additive effect, $i e$ the infinitesimal model (Wray and Hill, 1989; Dekkers, 1992). These deterministic methods invariably assume that the breeding values, phenotypes and selection criteria are normally distributed in the offspring generation, even after several generations of selection.

Bulmer (1980, p 153) argued that the departure from normality can be safely ignored following 1 generation of mass selection combined with random mating even when the heritability is 1 . Smith and Hammond (1987) investigated the departure from normality following 2 generations of mass selection combined with random mating. When heritability was 0.8 they showed that the error in calculating selection response assuming normality was $0.9,0.2$ and $-1.8 \%$ when 10,40 and $90 \%$, respectively, of progeny were retained for breeding (see their table III). The trend in the error was to underestimate response with intense selection and overestimate response when many progeny were retained for breeding. As heritability decreased the absolute error arising from the assumption of normality became even smaller.

Selection combined with positive assortative mating (hereafter called assortative mating) will increase the rate of genetic progress over that achieved with selection followed by random mating. This has been demonstrated in experimental studies with Drosophila (McBride and Robertson, 1963) and Tribolium (Wilson et al, 1965), in stochastic computer simulations (De Lange, 1974) and in deterministic computer simulations (Fernando and Gianola, 1986; Smith and Hammond, 1987; Tallis and Leppard, 1987).

Smith and Hammond (1987) used multivariate normal distribution theory to predict the advantage in selection response of assortative mating over random mating after 2 generations of mass selection. Their methodology accommodated both variance loss due to selection and the departure from normality in the offspring generation. They also investigated the advantage when a selection index, incorporating parental information, was used. They found that at low heritability, the advantage was much higher with index selection than with mass selection. Due to theoretical difficulties, Smith and Hammond (1987) were unable to consider more than 2 generations of selection.

Tallis and Leppard (1987) investigated the advantage at any generation of assortative mating over random mating under mass selection. However the model 
they proposed assumed normality in each offspring generation when predicting the expected genetic gain under truncation selection (see their equation [12]).

Smith and Hammond (1987) questioned the assumption of normality in the offspring generation when heritability was high and parents were mated assortatively. When heritability was 0.8 they showed that the error in assuming normality for the calculation of selection response following 2 generations of mass selection combined with assortative mating was $3.1,0.5$ and $-4.8 \%$ when 10,40 and $90 \%$ of progeny were retained for breeding (see their table III). As heritability decreased the absolute error arising from the assumption of normality became smaller.

Fernando and Gianola (1986) investigated the response to selection combined with assortative mating in two $N$-loci models. Model $A$ assumed 2 alleles per locus while Model $B$ assumed an infinite number of alleles per locus. In Model $B$ selection response was calculated assuming phenotype was normally distributed in each generation (see their equations [30-34]). However in Model $A$ the phenotypic distribution was allowed to be a mixture of normal distributions as parents were selected by truncation across $3^{N}$ genotype groups and were randomly mated in 3 mating groups which were formed on the basis of similarity of phenotype. A maximum of 3 loci were used in Model $A$.

A mixtures approach is also proposed in this paper, but the methodology is derived from open nucleus breeding theory assuming an infinitesimal model. James $(1989$, p 191) recognised the connection between multi-tier open nucleus breeding schemes and assortative mating programmes. This paper develops and evaluates this connection.

This paper proposes a deterministic model, which is used to predict the genetic gain at each generation when mating is assortative. The multi-tier model allows the distribution of progeny breeding values to be non-normal at each generation by considering it to be composed of a mixture (tiers) of normal distributions. The value of assortative mating is investigated deterministically when selection is either on individual phenotype or on best linear unbiased prediction (BLUP) estimates of breeding value (ebv) using an animal model. Stochastic simulation is used to evaluate the accuracy of the deterministic multi-tier model.

\section{MATERIALS AND METHODS}

The infinitesimal model is assumed in an infinite population with no accumulation of inbreeding. Selection is for a single trait with initial heritability $h^{2}$ before selection. When mating is random the joint distribution of breeding values and selection criteria are assumed multivariate normal at each generation before selection. The symbols $a$ and $b$ represent the proportions of all male and female offspring, respectively, used for breeding. Generations are assumed discrete.

\section{Multi-tier model concept}

Conceptually, assortative mating involves dividing the population into tiers with the best sire and best dam mated in the top tier, the next best pair (possibly the same sire) mated in the second tier, ...etc, and finally the worst selected sire and dam mated in the bottom tier. With an infinite population there would be an 
infinite number of tiers each of the same size, a single mating pair. With only a single mating pair in each tier it can be correctly assumed that mating within a tier is random.

To deterministically simulate assortative mating, the population is divided into $n$ tiers of equal size. Within each tier, mating is assumed random while the selection criterion is assumed normally distributed before selection. Parents are selected by truncation across tiers. The best proportion $(1 / n)$ of male and female parents are selected as tier 1 parents. The next best proportion $(1 / n)$ of male and female parents are selected as tier 2 parents and so on. This procedure of selecting across tiers and randomly mating within tiers is followed for the required number of generations.

As the number of tiers $(n)$ increases the population genetic gain per generation will tend toward an asymptote. This asymptote will be the deterministic prediction of the response to selection in conjunction with assortative mating. This procedure can be used to predict the response to selection combined with assortative mating at any generation.

The main issue is then the determination of the tier in which selected progeny are mated given their tier of birth. This issue is resolved using a selection and mating algorithm based on genetic groups as presented in the next section. The genetic groups are defined by 'tier of birth' and 'tier of mating' combinations. For example, with 3 tiers there are 9 genetic groups for each sex which have to be determined for each generation; 3 tiers of birth by 3 tiers in which mated (fig 1). With 50 tiers there are 2500 genetic groups for each sex which have to be determined for each generation. Determining genetic group composition is done separately for males and females.
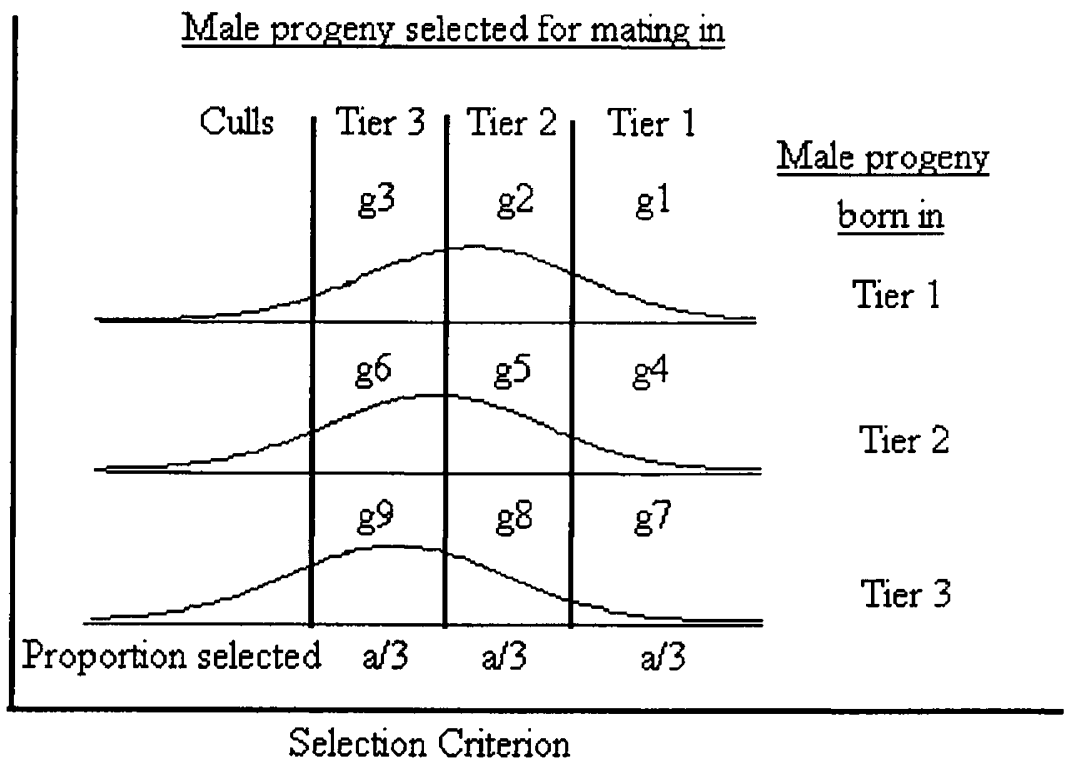

Fig 1. Diagrammatic representation of the 9 male genetic groups (g1, g2, .,g9) which have to be determined each generation in a deterministic 3-tier model of assortative mating following selection. 


\section{Deterministic selection and mating algorithm}

Animals are selected either on individual phenotype (mass selection) or on index ISD of Wray and Hill (1989) retaining those with either the largest phenotypic value or the largest index values as parents of the next generation. Selection is by truncation across the tiers. As detailed below the best in each tier are mated in the top tier. The next best in the second top tier, and so on. Within each tier, mating is random and the joint distribution of progeny phenotype, selection index and breeding value is assumed multivariate normal.

The index ISD uses records from the individual, its full and half sibs and the estimated breeding values of its sire, dam and all dams mated to its sire. This index is used to deterministically predict response when selection is based on breeding values estimated by a BLUP animal model. As not all relatives are used in the index, it is hereafter denoted nBLUP (nearly BLUP animal model).

For nBLUP selection, the EBVM (ebv selection and migration) method given by Shepherd and Kinghorn (1993) for 2-tier systems and Shepherd (1991) for 3-tier systems can be used without change to evaluate the response to selection using 2and 3-tier systems. The extension of the algorithm to $n$ tiers is quite straightforward and involves no new concepts. However extensive modifications are necessary to change various scalars into $n$ dimensional vectors and $n$ by $n$ dimensional matrices.

For mass selection, the EBVM algorithm described by Shepherd and Kinghorn (1992) for 3-tier open nucleus breeding systems can be used after slight modification. This is because selection in this algorithm is on ebv calculated as the regressed within-tier phenotypic deviation (rWTPD). That is, $e b v=\bar{P}_{t i e r}+h^{2}\left(P_{i}-\bar{P}_{\text {tier }}\right)$ where $P_{i}$ and $\bar{P}_{\text {tier }}$ are the phenotypic value of animal $i$ and the mean phenotypic value of all contemporary progeny in the same tier, respectively.

For mass selection this EBVM algorithm requires 2 modifications because the within-tier deviations are not regressed. That is, for mass selection $e b v=$ $\bar{P}_{\text {tier }}+\left(P_{i}-\bar{P}_{t i e r}\right)=P_{i}$. The modifications are: (1) replace ebv in steps 1-4 with phenotypic value; and (2) replace $\sigma_{I}$ in the identities for the standardised truncation points in steps $2-4$ with $\sigma_{A} / h\left(=\sigma_{P}\right)$, the phenotypic standard deviation. Now the EBVM method becomes the PM (phenotype selection and migration) method and is suitable for deterministically simulating mass selection followed by assortative mating.

The Appendix gives the PM method for $n$ tiers and also the deterministic Bulmer method of predicting genetic gain for random mating following mass selection. The deterministic methods used to model the joint effects of assortative mating and selection will hereafter be called the asymptotic PM method for mass selection and the asymptotic EBVM method for nBLUP selection. The adjective asymptotic emphasises that the prediction is made at a sufficiently large number of tiers such that the asymptote is reached.

In fact it usually took between 50 and 70 tiers before the response to selection reached its asymptote. This asymptote was sometimes reached in fewer tiers by using unequal tier sizes. In all cases examined the asymptotes using equal and unequal tier sizes were the same (as expected). Hence in reporting results no mention is made of relative tier size and usually between 50 and 70 tiers were used to determine the asymptote. 


\section{Stochastic simulation}

Stochastic simulations were carried out to check the deterministic predictions made by the asymptotic PM and asymptotic EBVM algorithms. These algorithms account for variance loss due to selection but as an infinite population is assumed no account is taken of variance loss due to inbreeding. Hence the stochastic simulations generate progeny breeding values without loss of within-family genetic variance due to parental inbreeding.

Initially a foundation population of $S$ sires and $D$ dams was created in which breeding values $A_{i}$ were randomly sampled from a normal distribution with mean zero and variance $\sigma_{A}^{2}=h^{2} \sigma_{P}^{2}$ where $\sigma_{P}^{2}$ was 1 . The unrelated foundation parents were randomly mated to produce the initial progeny crop for selection. Progeny breeding values were randomly sampled from a normal distribution with mean $0.5\left(A_{s}+A_{D}\right)$, the mean parental breeding value, and variance $0.5 \sigma_{A}^{2}$. Phenotypic values were simulated as $P_{i}=A_{i}+E_{i}$ where $E_{i}$ was randomly sampled from a normal distribution with mean zero and variance $\left(1-h^{2}\right) \sigma_{P}^{2}$.

A proportion $a$ of male progeny and $b$ of female progeny were retained for breeding each generation. Selection was either on individual phenotype or on BLUP ebv using an animal model (aBLUP). Parents were selected by truncation on the selection criterion. No fixed effects except the overall mean were included in the aBLUP evaluation. The calculation of the inverse of the numerator relationship matrix assumed no inbreeding as no progeny genetic variance was lost due to parental inbreeding. Each generation the system of linear equations for aBLUP was solved by Gauss-Seidel iteration. The iteration was stopped when $\sqrt{\sum\left(r_{i}-\widehat{r}_{i}\right)^{2} / \sum r_{i}^{2}}<1 \times 10^{-6}$ where $r_{i}$ and $\widehat{r}_{i}$ are the right-hand side of equation $i$ and the estimated right-hand side of equation $i$, respectively.

The animals selected for breeding were mated either randomly or assortatively. For assortative mating, sires and dams were ranked in descending order of either phenotype or aBLUP ebv to determine mates. The best sire was mated to the best $m_{1}$ dams, the next best sire was mated to the next best $m_{2}$ dams, and so on until all animals selected for breeding were allocated mates. Usually $m_{i}=b / a$ for each sire.

The total number of dams was 1000 with either 1,2 or $10(1 / b)$ progeny of each sex per dam. The number of dams mated to each sire was either 1,2 or 10 $(b / a)$. There were 500 replicates for mass selection, while for aBLUP selection the number of replicates was 400 and 200 for heritabilities 0.1 and 0.4 , respectively. The number of generations simulated was 10 and 5 for mass selection and aBLUP selection, respectively.

To simulate very low selection intensity in both males and females $(a=0.9, b=1)$ 900 sires were mated to 1000 dams with 1 male and 1 female offspring per dam. To achieve this mating ratio, 100 sires were randomly chosen for mating twice, while the remaining 800 sires were allocated only 1 mate. With assortative mating the number of mates allocated to a sire was taken into account following ranking on the selection criterion. There were 5000 replicates of this scheme for mass selection. 


\section{RESULTS AND DISCUSSION}

\section{Mass selection}

Table I shows the percent increase in genetic gain from generation 1 to 2 of the PM method (using between 10 and 50 tiers) over that achieved with random mating. As the number of tiers increased from 10 to 50 the predicted genetic gain from generation 1 to 2 tended to asymptote and hence so did the percent increase over random mating as shown in table I. For all selection intensities and heritabilities examined the percent increase was stable by 50 tiers. Hence the values in column $\% I_{50}$ (table I) are the deterministic predictions for the asymptotic PM method of the percent increase in genetic gain from generation 1 to 2 due to mating assortatively rather than randomly following mass selection. The trend for the PM method to asymptote as the number of tiers increased occurred at every generation as envisaged in the concept of the model.

The deterministic prediction of the advantage from generation 1 to 2 of assortative mating over random mating increased as heritability increased and as selection intensity decreased. Similar trends have been reported in the literature (Fernando and Gianola, 1986; Smith and Hammond, 1987).

Smith and Hammond (1987) gave exact theoretical results for the deterministic percent increase in genetic gain from generation 1 to 2 of assortative mating over random mating. The assumptions used in their evaluation were the same as those used in this evaluation, ie an infinite population and the infinitesimal model. However they allowed for non-normal progeny distributions when mating both randomly and assortatively. Their results are presented in column $\% I_{S H}$ in table I and are directly comparable with the results in column $\% I_{50}$. The discrepancy between the 2 columns as a percentage of $\% I_{S H}$ is given in column \%error.

When heritability is 0.1 , the asymptotic PM method slightly overestimates the advantage when selection intensity is high and tends to underestimate the advantage when selection intensity is low (table I). When heritability is 0.4 , the asymptotic PM method is once again quite accurate when approximately $50 \%$ of progeny are retained for breeding. However as selection intensity increases the asymptotic PM method overestimates the advantage, with the percentage error increasing with selection intensity. The opposite trend occurs as the proportion of progeny retained for breeding increases from 0.5. Namely, the asymptotic PM method underestimates the advantage, with the absolute percentage error increasing as selection intensity decreases.

The same general trends occur for heritability 0.8 as occur for the other heritabilities (table I). However the absolute magnitude of each percentage error when heritability is 0.8 is larger than the corresponding percentage error when heritability is smaller.

The reason for the discrepancies at high and low selection intensity was investigated by partitioning up the percent increase into its component parts. A heritability of 1 was chosen to maximise the discrepancies. Table II shows various deterministic predictions of genetic gain from generation 1 to 2 using either random or assortative mating.

The columns $\% I_{P M}$ and $\% I_{S H}$ (table II) show the percentage increase in genetic gain of assortative mating over random mating using the PM method and the 
Table I. Deterministic predictions of the percentage increase in genetic gain $\left(\% I_{n}\right)$ from generation 1 to 2 for the PM method with $n$ tiers compared with random mating following mass selection.

\begin{tabular}{|c|c|c|c|c|c|}
\hline \multirow[t]{2}{*}{ Proportion selected $(\mathrm{a}=\mathrm{b})$} & \multicolumn{4}{|c|}{ Percentage increase from generation 1 to 2} & \multirow[t]{2}{*}{$\%$ error } \\
\hline & $\% \mathrm{I}_{10}$ & $\% \mathrm{I}_{30}$ & $\% \mathrm{I}_{50}$ & $\% \mathrm{I}_{\mathrm{SH}}$ & \\
\hline \multicolumn{6}{|l|}{ Initial heritability $=0.1$} \\
\hline 0.1 & 0.88 & 0.90 & 0.90 & 0.89 & 0.9 \\
\hline 0.2 & 1.06 & 1.11 & 1.13 & 1.12 & 0.5 \\
\hline 0.4 & 1.53 & 1.55 & 1.56 & 1.55 & 0.3 \\
\hline 0.5 & 1.75 & 1.78 & 1.78 & 1.78 & 0 \\
\hline 0.6 & 2.00 & 2.03 & 2.03 & 2.04 & -0.5 \\
\hline 0.8 & 2.66 & 2.70 & 2.71 & 2.74 & -1.1 \\
\hline 0.9 & 3.18 & 3.25 & 3.25 & 3.30 & -1.5 \\
\hline \multicolumn{6}{|l|}{ Initial heritability $=0.4$} \\
\hline 0.1 & 4.15 & 4.24 & 4.25 & 4.01 & 6.0 \\
\hline 0.2 & 4.87 & 4.96 & 4.97 & 4.76 & 4.4 \\
\hline 0.4 & 5.99 & 6.07 & 6.08 & 6.00 & 1.3 \\
\hline 0.5 & 6.51 & 6.60 & 6.60 & 6.62 & 0.3 \\
\hline 0.6 & 7.05 & 7.15 & 7.15 & 7.29 & -1.9 \\
\hline 0.8 & 8.36 & 8.51 & 8.53 & 8.98 & -5.0 \\
\hline 0.9 & 9.53 & 9.65 & 9.66 & 10.32 & -6.4 \\
\hline \multicolumn{6}{|l|}{ Initial heritability $=0.8$} \\
\hline 0.1 & 9.42 & 9.61 & 9.62 & 8.63 & 11.5 \\
\hline 0.2 & 10.08 & 10.21 & 10.22 & 9.44 & 8.3 \\
\hline 0.4 & 10.77 & 10.88 & 10.89 & 10.65 & 2.3 \\
\hline 0.5 & 11.00 & 11.12 & 11.13 & 11.22 & -0.8 \\
\hline 0.6 & 11.19 & 11.33 & 11.34 & 11.82 & -4.1 \\
\hline 0.8 & 11.75 & 11.85 & 11.86 & 13.31 & -10.9 \\
\hline 0.9 & 12.28 & 12.46 & 12.47 & 14.48 & -13.9 \\
\hline
\end{tabular}

The same proportion of males $(a)$ and females $(b)$ are selected. $\% I_{S H}$ is the relevant percentage increase in table I of Smith and Hammond (1987). The column \%error is $100\left(\% I_{50}-\% I_{S H}\right) / \% I_{S H}$.

method of Smith and Hammond (1987), respectively. These columns show similar comparative trends to the corresponding columns in table $\mathrm{I}\left(\% I_{50}\right.$ and $\left.\% I_{S H}\right)$. The percent increase predicted by the asymptotic PM method overestimates the value of assortative mating when selection is intense and underestimates the value when a large proportion of progeny are retained for breeding.

For assortative mating the predictions of genetic gain in table II were practically identical for the asymptotic PM method and for the method of Smith and Hammond (1987). The maximum percentage error was less than $0.03 \%$. Hence the cause of the discrepancies in the percent increase predictions was due to the discrepancies in the deterministic predictions of genetic gain with random mating. The column \%error shows that for random mating the Bulmer prediction $\left(G_{B}\right)$ underestimated $G_{S H}^{r}$ when selection was intense and overestimated $G_{S H}^{r}$ when many progeny were 
Table II. Deterministic predictions of genetic gain $(G)$ from generation 1 to 2 for random and assortative mating following mass selection when heritability is 1 .

\begin{tabular}{lccccccr}
\hline $\begin{array}{l}\text { Proportion } \\
\text { selected }(\mathrm{a}=\mathrm{b})\end{array}$ & \multicolumn{3}{c}{ Random mating } & \multicolumn{3}{c}{ Assortative mating } & \multicolumn{2}{c}{$\begin{array}{c}\text { Percentage increase } \\
\text { from generation 1 to 2 } \\
\text { \% }\end{array}$} \\
& $\mathrm{G}_{\mathrm{B}}$ & $\mathrm{G}_{\mathrm{SH}}^{\mathrm{r}}$ & \%error & $\mathrm{G}_{\mathrm{PM}}$ & $\mathrm{G}_{\mathrm{SH}}^{a}$ & & \% $_{\mathrm{SH}}$ \\
\hline 0.05 & 1.556 & 1.575 & -1.2 & 1.7233 & 1.7230 & 10.73 & 9.39 \\
0.2 & 1.093 & 1.103 & -0.9 & 1.2184 & 1.2184 & 11.50 & 10.50 \\
0.5 & 0.659 & 0.658 & 0.1 & 0.7372 & 0.7373 & 11.92 & 12.05 \\
0.8 & 0.311 & 0.307 & 1.5 & 0.3491 & 0.3492 & 12.14 & 13.89 \\
0.95 & 0.103 & 0.101 & 2.2 & 0.1170 & 0.1170 & 13.23 & 15.86 \\
\hline
\end{tabular}

$G_{S H}$ predictions use the theory of Smith and Hammond (1987) for random $\left(G_{S H}^{r}\right)$ and assortative $\left(G_{S H}^{a}\right)$ mating. $G_{P M}$ and $G_{B}$ are the predicted genetic gain using the asymptotic PM method and Bulmer's method, respectively (Appendix). Columns $\% I_{S H}$ and $\% I_{P M}$ are equal to $100\left(G_{S H}^{a}-G_{S H}^{r}\right) / G_{S H}^{r}$ and $100\left(G_{\mathrm{PM}}-G_{\mathrm{B}}\right) / G_{\mathrm{B}}$ respectively. Column \%error is equal to $100\left(G_{\mathrm{B}}-G_{S H}^{r}\right) / G_{S H}^{r}$.

retained for breeding. These results agree with the findings reported by Smith and Hammond (1987).

Smith and Hammond (1987) were unable to extend their theory for assortative mating beyond 2 generations of selection. Hence to examine the performance of the asymptotic PM method beyond 2 generations of selection, stochastic simulation was used. Figure 2 shows the genetic gain at each of 10 generations for both random and assortative mating using low $(a=0.9, b=1)$, intermediate $(a=0.5, b=0.5)$ and high $(a=0.01, b=0.1)$ intensities of selection.

For random mating the deterministic prediction at each generation underestimated the stochastic genetic gain when selection was intense (fig $2 \mathrm{~A}$ ) and overestimated the stochastic genetic gain when selection intensity was low (fig $2 \mathrm{E}$ ). When $50 \%$ of progeny were retained for breeding (fig $2 \mathrm{C}$ ) the percentage error was much reduced. These trends agree with the findings of Smith and Hammond (1987) for generation 2 . The interesting result here is that the discrepancy at later generations is of a similar magnitude to that at generation 2. At generation 2 the percentage error was 1.2 and $0.7 \%$ for figures $2 \mathrm{~A}$ and $2 \mathrm{E}$, respectively. Averaged over all generations the percentage error was 0.8 and $0.9 \%$ for figures $2 \mathrm{~A}$ and $2 \mathrm{E}$, respectively. The discrepancy at generation 1 was $0.2 \%$ or less, in general agreement with Bulmer (1980) who found a percentage error of $0.15 \%$ in his deterministic example with a heritability of 1 .

For assortative mating combined with intense selection, the asymptotic PM method overestimated selection response significantly $(P<0.05)$ at all generations by a similar amount (fig $2 \mathrm{~B}$ ). The selection response was overestimated by $0.8 \%$ at generation 2 and by $0.6 \%$ averaged over all generations. This result does not concur with the findings of table II in which the asymptotic PM method agreed with the deterministic predictions of Smith and Hammond (1987). One possible explanation may be that a stochastic simulation with 50 sires may not be large enough to produce the infinite population result for assortative mating in this case. 

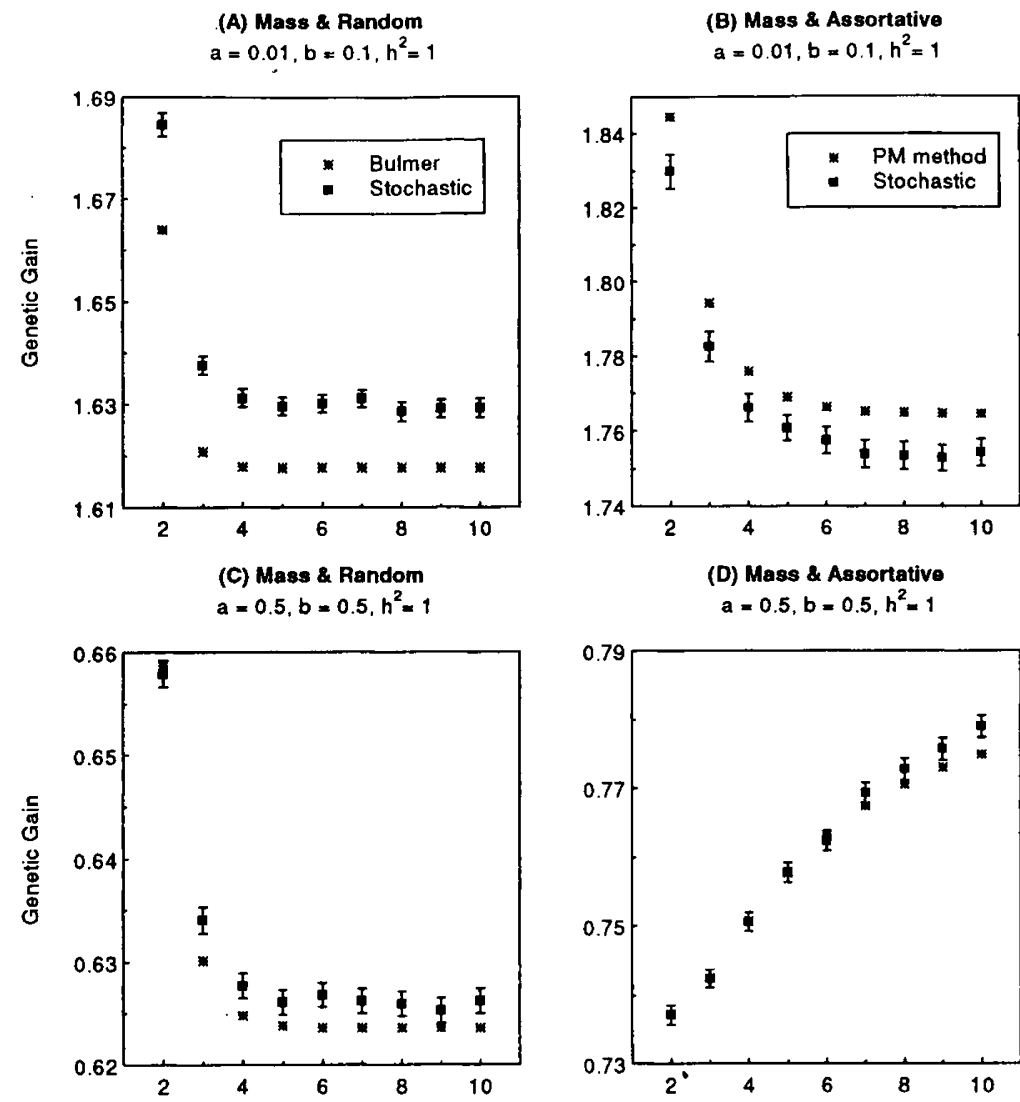

(D) Mass \& Assortative

(C) Mass \& Random

$a=0.5, b=0.5, h^{2}=1$

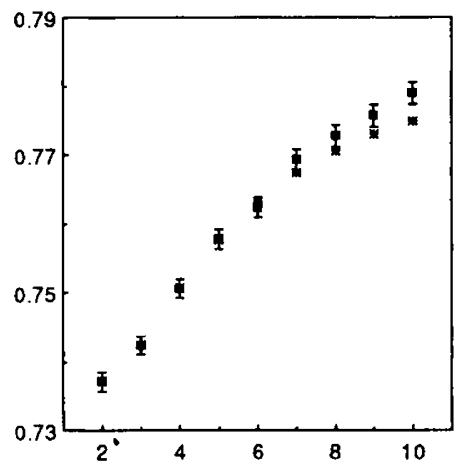

(E) Mass \& Random

$a=0.9, b=1, h^{2}=1$

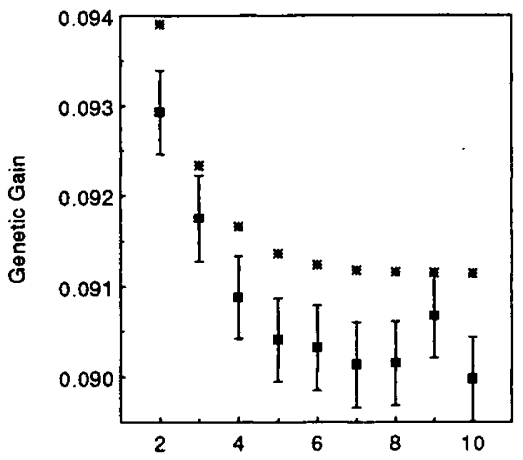

(F) Mass \& Assortative

$a=0.9, b=1, h^{2}=1$

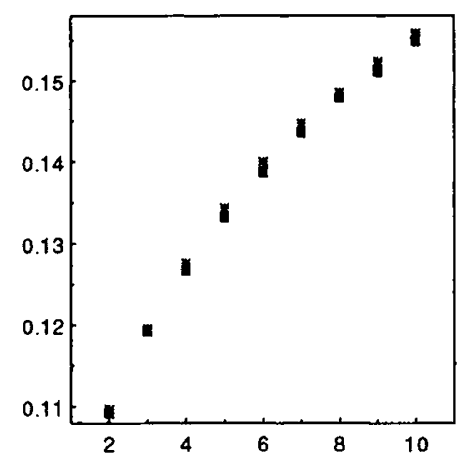

Generation Number

Generation Number

Fig 2. Stochastic simulation and deterministic prediction of the genetic gain at each of 10 generations for random and assortative mating following mass selection. The proportion of males and females retained for breeding are $a$ and $b$ respectively. The initial heritability is $h^{2}$. The error bars on the stochastic means give $95 \%$ confidence intervals. 
For the intermediate selection intensity in combination with assortative mating (fig 2D), the stochastic and deterministic predictions only differed significantly $(P<0.05)$ from generations 7 to 10 . Over these generations the average percentage error was less than $0.3 \%$.

For the low selection intensity in combination with assortative mating (fig $2 \mathrm{~F}$ ), the stochastic and deterministic predictions were significantly different $(P<0.05)$ from generations 4 to 10 . The average percentage error was $0.7 \%$ over these generations. The trend was for the deterministic prediction to overestimate the stochastic value.

Hence the main finding seems to be that the asymptotic PM method is a good predictor of genetic gain when assortative mating is used. There appears to be no error when compared to exact deterministic predictions for 2 generations. However stochastic simulations are often overestimated, possibly indicating that larger stochastic populations are needed for closer agreement with deterministic infinite population theory. In any case the percentage errors arising with the asymptotic PM method in the stochastic simulations were usually smaller in absolute magnitude than those found with the usual Bulmer procedure (fig 2).

Some interesting features of assortative mating can be easily demonstrated using the asymptotic PM method. The asymptotic PM method can indeed handle the non-normality induced by assortative mating. For $a=b=0.5$, Tallis and Leppard (1987, table I) found a percentage increase in genetic gain at equilibrium of assortative mating over random mating of $13.4 \%$ when heritability was 1 . Using figures $2 \mathrm{C}$ and $2 \mathrm{D}$ the percentage increase at generation 10 is 24.4 and $24.3 \%$ for the stochastic simulation and the asymptotic PM method, respectively. Figure $2 \mathrm{D}$ also shows that the genetic gain with assortative mating is still increasing at generation 10 , resulting in a percentage increase at equilibrium which will be even larger. Hence Tallis and Leppard's method of assuming normality in the offspring generation greatly underestimates the value of assortative mating in this case.

For $a=b=0.5$, Tallis and Leppard (table I, 1987) found percentage increases in genetic gain at equilibrium of assortative mating over random mating of 5.5, 8.9 and $12.8 \%$ for heritabilities of $0.2,0.4$ and 0.8 , respectively. The asymptotic PM method produces percentage increases at equilibrium of $6.1,11.2$ and $22.0 \%$ for heritabilities of $0.2,0.4$ and 0.8 , respectively. There is only a small difference between the 2 predictions when heritability is low, indicating that the normal approximation is reasonable in this case. However as heritability increases the difference gets progressively larger. This is expected as the distribution of breeding value becomes more non-normal as heritability increases. Hence assuming normality can greatly underestimate the value of assortative mating when heritability is high or when selection is not intense.

A feature that does not seem to have been reported in the literature is the difference between the percentage improvements of assortative mating over random mating at generations 2 and 10. Using the asymptotic PM method with a heritability of 0.1 , the ratio of the percentage improvement at generation 2 to that at generation 10 is $0.61,0.54$ and 0.46 for the proportions selected of $0.01,0.5$ and 0.9 , respectively. The trend is for the ratio to decrease as the intensity of selection decreases and it is caused by the proportionally larger increase in the percentage improvement at generation 10 as selection intensity decreases. 
This trend is even more pronounced at higher heritability indicating an even larger proportional increase in the percentage improvement at generation 10 as the intensity of selection decreases. For a heritability of 1 , the ratio of the percentage increase at generation 2 to that at generation 10 is $1.43,0.49$ and 0.26 for proportions selected of $0.01,0.5$ and 0.9 , respectively. When $a=b=0.9$, the percentage improvement in genetic gain is 12.6 and $48.8 \%$ at generations 2 and 10, respectively. Hence when heritability is high and selection intensity is low, assortative mating generates a lot of between-tier genetic variance but it takes many generations to produce (see figure $2 \mathrm{~F}$ ).

For intense selection and high heritability the advantage of assortative mating can be larger in the early generations. For example, when $a=b=0.01$ and heritability is 1 , the percentage improvements in genetic gain are 9.3 and $6.5 \%$ at generations 2 and 10, respectively. A similar result occurs in the stochastic simulations in figures $2 \mathrm{~A}$ and $2 \mathrm{~B}(8.6$ and $7.7 \%$ at generations 2 and 10$)$. The reason for this result seems to be related to that of a similar result found when investigating the effects of variance loss on the percentage improvement in genetic gain of 2-tier open nucleus breeding schemes over closed nucleus schemes (Shepherd, 1991). High heritability and intense selection cause a large variance loss very quickly in a random mating population (fig $2 \mathrm{~A}$ ). However a large amount (because heritability is high) of between-tier variance is generated very quickly (because selection is intense) by assortative mating under the same conditions (fig $2 \mathrm{~B}$ ). As the generations progress some of this between-tier variance is lost due to selection. These processes result in the percentage increase in genetic gain at generation 2 being larger than that at generation 10 .

Table III gives deterministic predictions of the percentage increase in genetic gain at generation 10 of assortative mating over random mating for various selection intensities and heritabilities. As found earlier when $a=b$ the main result is that the percentage increase at generation 10 of assortative mating over random mating increases as either the intensity of selection decreases or as heritability increases. As shown in table I and figure 2 these deterministic predictions are likely to overestimate the value of assortative mating with high selection intensity and underestimate the value with low selection intensity. The \%error values in table I can be used to give more accurate predictions, assuming the same \%error values occur at generation 10 . Table III also gives the ratio of the percentage increase at generation 2 and to that at generation 10 .

\section{BLUP selection}

Table IV gives deterministic predictions of the percentage increase in genetic gain at various generations for assortative mating over random mating. The PM method is used for mass selection while the EBVM method is used for nBLUP selection. In both prediction methods 50 equal sized tiers were used. Predictions were also calculated at equilibrium when the genetic gain with assortative mating had stabilised. As selection intensity decreased, the number of generations required to reach equilibrium increased (as indicated in fig 2). 
Table III. Deterministic predictions for mass selection of the percentage increase in genetic gain at generation 10 of assortative mating over random mating.

\begin{tabular}{|c|c|c|c|c|}
\hline \multirow[t]{2}{*}{ Initial heritability } & \multirow[t]{2}{*}{ Male Proportion (a) } & \multicolumn{3}{|c|}{ Proportion females selected (b) } \\
\hline & & 0.1 & 0.5 & 0.9 \\
\hline 0.1 & $\begin{array}{l}0.1 \\
0.5 \\
0.9\end{array}$ & $1.5(0.59)$ & $\begin{array}{l}2.2(0.57) \\
3.3(0.54)\end{array}$ & $\begin{array}{l}3.2(0.54) \\
4.7(0.51) \\
7.0(0.46)\end{array}$ \\
\hline 0.4 & $\begin{array}{l}0.1 \\
0.5 \\
0.9\end{array}$ & $5.4(0.79)$ & $\begin{array}{r}8.1(0.69) \\
11.4(0.89)\end{array}$ & $\begin{array}{l}12.7(0.58) \\
17.8(0.47) \\
27.3(0.35)\end{array}$ \\
\hline 0.8 & $\begin{array}{l}0.1 \\
0.5 \\
0.9\end{array}$ & $9.9(0.96)$ & $\begin{array}{l}15.4(0.74) \\
21.5(0.52)\end{array}$ & $\begin{array}{l}24.0(0.58) \\
34.0(0.39) \\
46.4(0.27)\end{array}$ \\
\hline 1.0 & $\begin{array}{l}0.1 \\
0.5 \\
0.9\end{array}$ & $11.4(0.97)$ & $\begin{array}{l}17.7(0.73) \\
24.3(0.49)\end{array}$ & $\begin{array}{l}26.7(0.57) \\
37.1(0.37) \\
48.8(0.26)\end{array}$ \\
\hline
\end{tabular}

In brackets is the ratio of the percent increase at generation 2 to that at generation 10 . Seventy tiers were used when heritability was 1 while 50 tiers were used for all other heritabilities

For each intensity of nBLUP selection the percentage increase in genetic gain gets larger as initial heritability increases (table IV). This effect mainly occurs in early generations and is largest at generation 2 when family information is smallest $(m=1, n p=2)$. As the generations pass, the difference in the percentage increase between heritabilities gets smaller until at equilibrium the percentage increase of assortative over random mating is independent of both initial heritability and the amount of family information (table IV). Being independent of initial heritability the percentage increase for nBLUP is identical to the deterministic prediction for mass selection at equilibrium when initial heritability is 1 (table IV). Hence at equilibrium the percentage increase for nBLUP depends solely on the intensity of selection.

A similar finding of independence in the advantage of opening a closed nucleus was reported for a deterministic model of BLUP selection in 2-tier open nucleus breeding schemes (Shepherd and Kinghorn, 1993). They used the EBVM algorithm with 2-tiers. Shepherd and Kinghorn (1993) discussed at length the reason for their finding. The same reasons are applicable when an infinite number of tiers are used with the EBVM method, ie assortative mating. Their conclusion was that it was basically caused by the between-tier analogy of the within-tier result reported by Dekkers (1992) for selection on BLUP ebv.

For nBLUP selection the percentage increase in genetic gain of assortative mating over random mating will be independent of initial heritability and family structure at any generation if there is sufficient pedigree history before selection commences. The influence of the amount of family information is shown in table IV. For example, if $a=b=0.1$ then for $m=1$ and $n p=2$ the percentage increase at generation 2 
Table IV. Deterministic predictions for mass and nBLUP selection of the percentage increase in genetic gain at various generations of assortative mating over random mating.

\begin{tabular}{|c|c|c|c|c|c|}
\hline \multirow[t]{2}{*}{ Proportion selected $(\mathrm{a}=\mathrm{b})$} & \multirow[t]{2}{*}{ Initial heritability } & \multicolumn{4}{|c|}{$\begin{array}{c}\text { Percentage increase in genetic gain } \\
\text { at generation }\end{array}$} \\
\hline & & 2 & 5 & 10 & Equilibrium \\
\hline \multicolumn{6}{|l|}{ Mass selection } \\
\hline 0.1 & 1.0 & 11.00 & 11.36 & 11.03 & 11.03 \\
\hline 0.5 & 1.0 & 11.92 & 21.32 & 23.82 & 24.49 \\
\hline 0.9 & 1.0 & 12.58 & 34.42 & 48.75 & $66.13^{*}$ \\
\hline \multicolumn{6}{|c|}{$n B L U P$ selection, $m=1, n p=2$} \\
\hline \multirow[t]{2}{*}{0.1} & 0.1 & 8.68 & 11.15 & 11.03 & 11.03 \\
\hline & 0.4 & 10.34 & 11.35 & 11.03 & 11.03 \\
\hline \multirow[t]{2}{*}{0.5} & 0.1 & 10.07 & 20.39 & 23.65 & 24.49 \\
\hline & 0.4 & 11.41 & 21.18 & 23.79 & 24.49 \\
\hline \multirow[t]{2}{*}{0.9} & 0.1 & 11.32 & 32.70 & 47.99 & $66.13^{*}$ \\
\hline & 0.4 & 12.25 & 34.14 & 48.63 & $66.13^{*}$ \\
\hline \multicolumn{6}{|c|}{$n B L U P$ selection, $m=50, n p=100$} \\
\hline \multirow[t]{2}{*}{0.1} & 0.1 & 10.57 & 11.36 & 11.03 & 11.03 \\
\hline & 0.4 & 10.98 & 11.36 & 11.03 & 11.03 \\
\hline \multirow[t]{2}{*}{0.5} & 0.1 & 11.59 & 21.26 & 23.80 & 24.49 \\
\hline & 0.4 & 11.90 & 21.32 & 23.82 & 24.49 \\
\hline \multirow[t]{2}{*}{0.9} & 0.1 & 12.37 & 34.30 & 48.69 & $66.13^{*}$ \\
\hline & 0.4 & 12.57 & 34.42 & 48.74 & $66.13^{*}$ \\
\hline
\end{tabular}

For nBLUP, $m$ and $n p$ denote the number of dams per sire and the number of progeny per dam respectively. Fifty equal-sized tiers were used in the PM and EBVM methods. ${ }^{*}$ Percentage increase at generation 100 using only 20 tiers.

is 8.68 and $10.34 \%$ for heritabilities 0.1 and 0.4 , respectively, whereas for $m=50$ and $n p=100$ the percentage increase at generation 2 is 10.57 and $10.98 \%$ for heritabilities 0.1 and 0.4 , respectively. With ample pedigree history the percentage increase at generation 2 for any heritability with nBLUP will be identical to that of mass selection at generation 2 for heritability 1 .

If there is no pedigree history when selection commences then the results for nBLUP will show similar trends to those found for mass selection in the early generations. For example for nBLUP selection with $m=1, n p=2$ and $a=b=0.1$, the percentage increases at generation 2 are $8.68,10.34$ and $11.00 \%$ for heritabilities of $0.1,0.4$ and 1 , respectively, when no pedigrees are known before selection commences. For mass selection, the percentage increase at generation 2 is 0.89 , 4.01 and $11.00 \%$ for heritabilities of $0.1,0.4$ and 1 , respectively. Hence, when there is no pedigree history before selection commences the advantage of assortative mating over random mating at generation 2 increases with heritability for nBLUP. However the percentage increase for low heritability is much larger with nBLUP than with mass selection. These trends were also found by Smith and Hammond (1987) in generation 2 for selection on an index, which included individual and parental phenotypic information (see table IV). For $a=b=0.1$, they calculated 
percentage increases of 5.60 and $7.23 \%$ for heritabilities 0.1 and 0.4 , respectively, when selecting on the index.

The deterministic prediction of nBLUP was compared with stochastic simulation for 2 selection intensities applied for 5 generations. Figure 3 gives results for $a=0.05$ and $b=0.5$ (high selection intensity), while figure 4 gives results for $a=0.5$ and $b=1$ (intermediate selection intensity). In both figures heritabilities of 0.1 and 0.4 were used.

With random mating the deterministic method of Wray and Hill (1989) tended to underestimate the stochastic genetic gain when the selection intensity was high, being significantly different $(P<0.05)$ at 3 of the 4 generations when heritability was 0.1 (fig $3 \mathrm{~A}$ ). The percentage error was $2.1 \%$ averaged over the 4 generations. When heritability was 0.4 only 2 of the differences were statistically significant (fig $3 \mathrm{C}$ ), while the percentage error averaged only $0.4 \%$. Only 1 of the 8 deterministic predictions was statistically significant at the intermediate selection intensity (fig $4 \mathrm{~A}$ and $4 \mathrm{C}$ ). In general terms these results tended to agree with the earlier findings for mass selection at high heritability: underestimation at high selection intensity and better accuracy at intermediate selection intensity.

With assortative mating the asymptotic EBVM method tended to overestimate the stochastic genetic gain when the selection intensity was high, with the overestimation increasing as generations passed (fig 3B and 3D). At generation 5 the overestimation was 3.4 and $3.3 \%$ for heritabilities 0.1 and 0.4 , respectively. This result is unlikely to be a finite population effect as 100 sires and 1000 dams were mated each generation. Only 1 of the 8 deterministic predictions was statistically significant at the intermediate selection intensity (fig 4B and 4D).

For high selection intensity, the trend for the asymptotic EBVM method to overestimate, and for the Wray and Hill method to underestimate, genetic gain results in overestimates of the advantage of assortative mating over random mating. For example, in figures $3 \mathrm{~A}$ and $3 \mathrm{~B}$ the deterministic percentage increase of assortative mating over random mating is $10.5,14.6,15.6$ and $15.8 \%$ for generations $2,3,4$ and 5 , respectively, whereas the stochastic mean percentage increase is 6.8 , $10.8,10.4$ and $10.8 \%$. At the intermediate selection intensity the agreement between the percentage increases is good.

The deterministic finding of the independence with respect to heritability of the equilibrium advantage of assortative mating over random mating is evident in the stochastic simulation. For $a=0.05$ and $b=0.5$, the mean stochastic percentage increases at generation 5 are $10.8 \%$ and $11.8 \%$ for heritabilities 0.1 and 0.4 , respectively, whereas the deterministic predictions are 15.8 and $16.0 \%$. For $a=0.5$ and $b=1$, the mean stochastic percentage increases at generation 5 are 33.6 and $36.6 \%$ for heritabilities 0.1 and 0.4 , respectively, whereas the deterministic predictions are 34.5 and $35.9 \%$. The closer agreement with the more intense selection is expected as equilibrium is reached quicker when selection is more intense.

Hence the deterministic finding of the independence with respect to heritability and family structure of the equilibrium advantage of assortative mating over random mating seems to be a feature of BLUP in large populations. In view of this finding table III with a heritability of 1 can be used to give predictions of the percentage increase at generation 10 for BLUP. However similar corrections as discussed for mass selection are needed to reduce these predictions when selection is intense. 
(A) BLUP \& Random

$a=0.05, b=0.5, h^{2}=0.1$

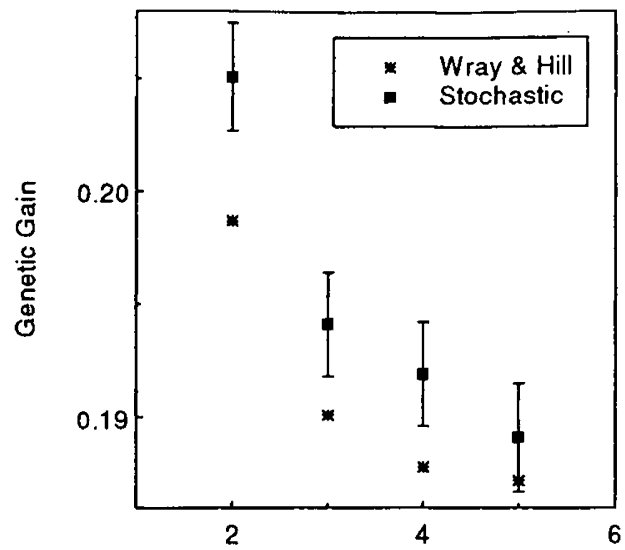

(C) BLUP \& Random

$a=0.05, b=0.5, h^{2}=0.4$

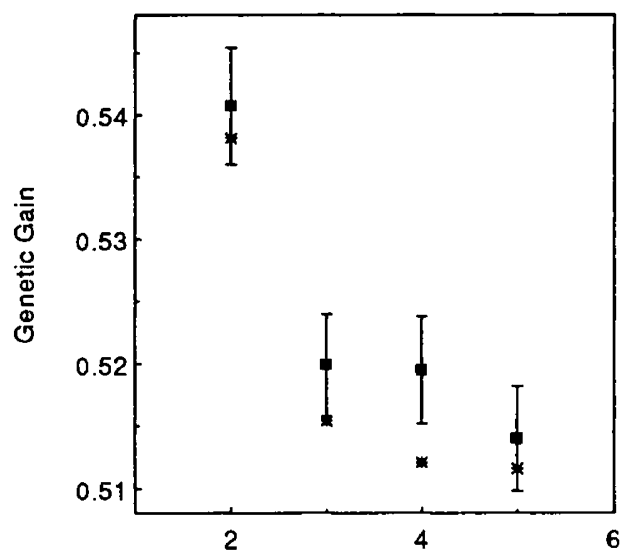

Generation Number
(B) BLUP \& Assortative

$a=0.05, b=0.5, h^{2}=0.1$

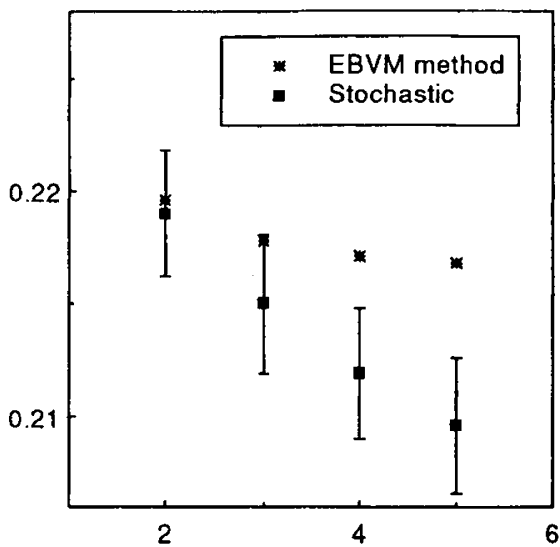

(D) BLUP \& Assortative

$a=0.05, b=0.5, h^{2}=0.4$

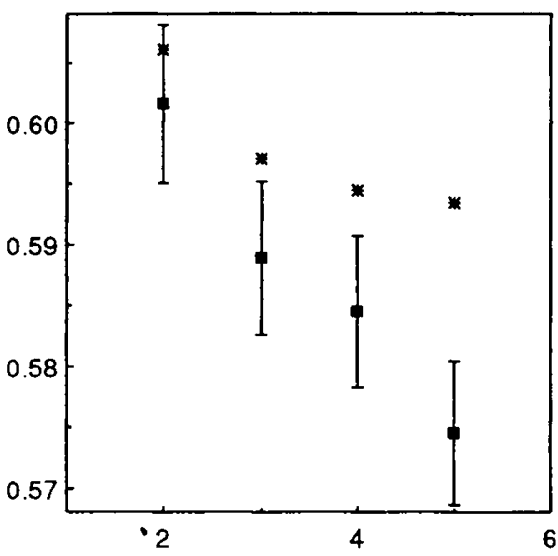

Generation Number

Fig 3. Stochastic simulation and deterministic prediction of the genetic gain at each of 5 generations for random and assortative mating following BLUP selection. The proportion of males and females retained for breeding are $0.05(a)$ and $0.5(b)$, respectively. The initial heritability is $h^{2}$. The error bars on the stochastic means give $95 \%$ confidence intervals.

A more comprehensive stochastic simulation is needed to evaluate the adequacy of the asymptotic EBVM method when selection is on BLUP ebv. The effects of population size and the number of sires also need to be investigated by stochastic simulation for both the PM and EBVM methods. 
(A) BLUP \& Random

$a=0.5, b=1, h^{2}=0.1$

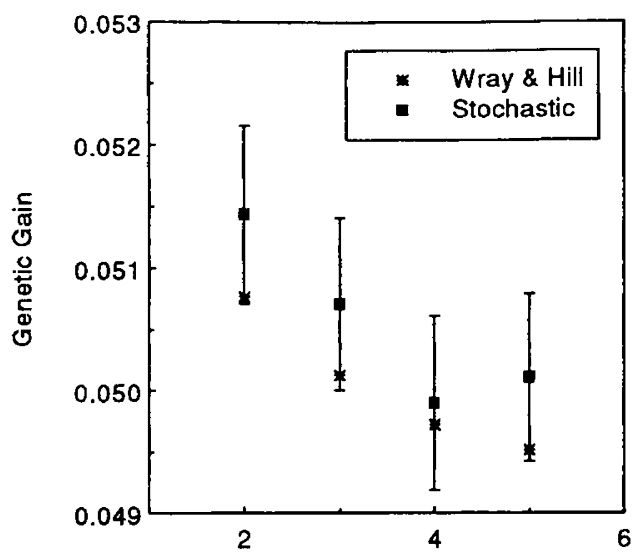

(C) BLUP \& Random

$a=0.5, b=1, h^{2}=0.4$

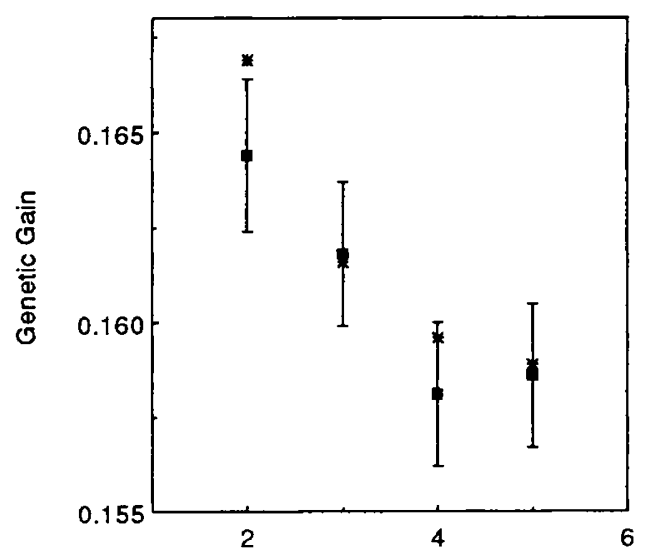

Generation Number
(B) BLUP \& Assortative

$\mathrm{a}=0.5, \mathrm{~b}=1, \mathrm{~h}^{2}=0.1$

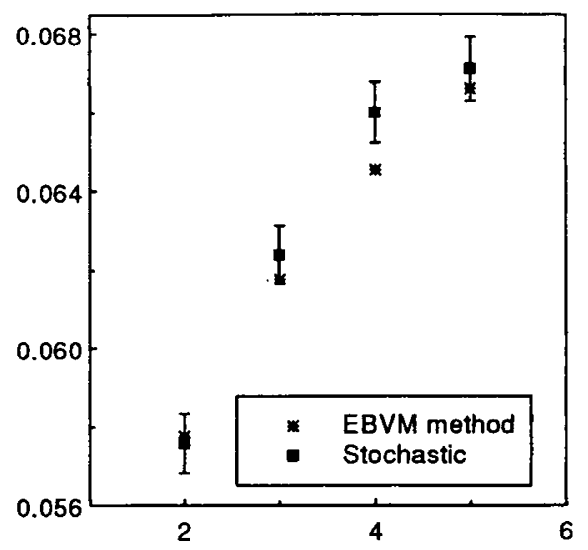

(D) BLUP \& Assortative

$$
a=0.5, b=1, h^{2}=0.4
$$

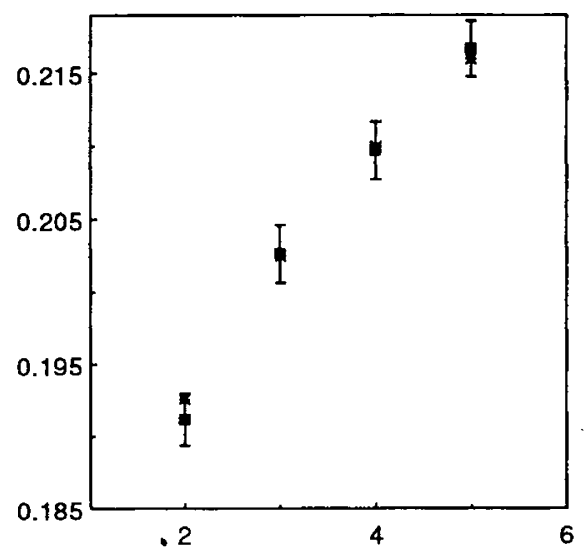

Generation Number

Fig 4. Stochastic simulation and deterministic prediction of the genetic gain at each of 5 generations for random and assortative mating following BLUP selection. The proportion of males and females retained for breeding are $0.5(a)$ and $1 .(b)$, respectively. The initial heritability is $h^{2}$. The error bars on the stochastic means give $95 \%$ confidence intervals. 


\section{CONCLUSION}

\section{Selection history}

A recurring issue in the recent history of animal breeding theory is the importance of incorporating selection history into selection strategies for populations when generations overlap. This was first demonstrated by Bichard et al (1973) and later extended by Hopkins and James (1977) in their Progeny Selection and Parent Selection strategies. It is now well known that a BLUP animal model takes account of the accumulated selection history, not of age groups as in the methods of Hopkins and James (1977) but of individuals by using the ancestral information in the numerator relationship matrix.

Assortative mating, whether between-tier as in open nucleus breeding systems or within-tier, involves spreading the population in terms of genetic merit and can be considered as the formation of genetic groups based on mating pairs or mating groups. When generations overlap a similar spread occurs due to the formation of genetic groups based on age and selection response can be increased if the spread or history is taken into account. Both between-tier and within-tier assortative mating take selection history into account and thus increase the response to selection.

The proposed model of positive assortative mating following selection accounts for selection history by following the accumulated selection differentials of individual tiers, which in the limit become mating pairs. In doing this the model allows the population distribution of breeding values to be a mixture of normal distributions. This feature produces more accurate predictions of the advantage of assortative mating over random mating than a model assuming normality in the offspring generation, eg, the model of Tallis and Leppard (1987).

\section{BLUP selection}

The advantage of assortative mating over random mating is higher for BLUP than for mass selection as BLUP can more accurately predict breeding values. Thus mate allocation will more closely reflect pairing on true breeding values and hence produce more genetic spread in the population.

Mass selection does not regress an individual's within-tier phenotypic deviation as is done with rWTPD selection in open nucleus breeding systems (Shepherd and Kinghorn, 1993). Hence for low heritability the pairing of mates with mass selection is a poor reflection of pairing on true breeding values, resulting in a small advantage to assortative mating. However, as heritability increases, the accuracy of pairing increases with mass selection and results in more advantages for assortative mating.

An interesting finding with BLUP selection is that the advantage of assortative mating over random mating is independent of initial heritability and family information at any generation if there is ample pedigree history available before selection commences. Stochastic simulation showed this trend in populations of 1000 dams and at least 100 sires. Shepherd (1991) showed that this independence was not solely a property of BLUP, but is in fact a feature of ancestral regression, such that it holds for the nBLUP index used here. 
The value of assortative mating at low to moderate heritability is greatly increased when BLUP selection is used rather than mass selection. Smith and Hammond (1987) found a similar result in generation 2 using a parental index. This feature of BLUP certainly makes assortative mating an attractive option for breeders wishing to increase the rate of genetic gain.

However, it is important to remember that the rate of inbreeding will also increase under assortative mating (McBride and Robertson, 1963) and no account of genetic variance loss due to the accumulation of inbreeding has been made in this paper. Under reasonably intense selection the increased BLUP genetic gains from assortative mating may well be cancelled out by the loss of genetic variance due to inbreeding, particularly at low heritability as the ebvs of relatives will be more highly correlated giving more co-selection of relatives. This will certainly be true in the longer term for small populations. Deterministic methods of predicting rates of inbreeding with assortative mating are desirable but are likely to be complex given the findings for randomly mated populations (Wray and Thompson, 1990).

\section{ACKNOWLEDGMENTS}

Part of this work was carried out at the University of New England while RKS held an Australian Wool Corporation Postgraduate Scholarship and was on study leave from the Queensland Department of Primary Industries. Both N Wray and S Smith are thanked for providing copies of their deterministic computer programs.

\section{REFERENCES}

Bichard M, Pease AHR, Swales PH, Ozkutuk K (1973) Selection in a population with overlapping generations. Anim Prod 17, 215-227

Bulmer MG (1980) The Mathematical Theory of Quantitative Genetics. Clarendon Press, Oxford, UK

De Lange AO (1974) A simulation study of the effects of assortative mating on the response to selection. In: 1st World Congress on Genetics Applied to Livestock Production, Madrid, Spain, Vol 3, 421-435

Dekkers JCM (1992) Asymptotic response to selection on best linear unbiased predictors of breeding values. Anim Prod 54, 351-360

Fernando RL, Gianola D (1986) Effect of assortative mating on genetic change due to selection. Theor Appl Genet 72, 395-404

Hopkins IR, James JW (1977) Some optimum selection strategies and age structures with overlapping generations. Anim Prod 25, 111-132

James JW (1989) Design of animal breeding programmes. In: Evolution and Animal Breeding. Reviews on Molecular and Quantitative Approaches in Honour of Alan Robertson (WG Hill, TFC Mackay, eds), CAB International, Wallingford, UK, 189194

McBride G, Robertson A (1963) Selection using assortative mating in Drosophila melanogaster. Genet Res 4, 356-369

Shepherd RK (1991) Multi-tier open nucleus breeding schemes. PhD thesis, University of New England, Armidale, Australia

Shepherd RK, Kinghorn BP (1992) Optimising multi-tier open nucleus breeding schemes. Theor Appl Genet 85, 372-378 
Shepherd RK, Kinghorn BP (1993) A deterministic model of BLUP selection in two-tier open nucleus breeding schemes. Livest Prod Sci 33, 341-354

Smith SP, Hammond K (1987) Assortative mating and artificial selection: a second appraisal. Genet Sel Evol 19, 181-196

Tallis GM, Leppard P (1987) The joint effects of selection and assortative mating on a single polygenic character. Theor Appl Genet 75, 41-45

Wilson SP, Kyle WH, Bell AE (1965) The effects of mating systems and selection on pupa weight in Tribolium. Genet Res 6, 341-351

Wray NR, Hill WG (1989) Asymptotic rates of response from index selection. Anim Prod $49,217-227$

Wray NR, Thompson R (1990) Methods of predicting rates of inbreeding in selected populations. Theor Appl Genet 80, 503-512

\section{APPENDIX: DETERMINISTIC METHODS FOR MASS SELECTION}

\section{Assortative mating - the PM method for $n$ tiers}

The following algorithm describes the PM method for $n$ tiers with a proportion $p_{i}$ of the population in tier $i$. Usually $p_{i}=1 / n$.

\section{Step 1}

Using known genetic means and variances, line up the assumed normal distributions of progeny phenotype in each tier using the same abscissa scale.

\section{Step 2}

Select as dams for tier 1 all female progeny whose phenotype is larger than a common truncation point $t_{1}$ which is chosen such that the best $b p_{1}$ of all female progeny become tier 1 dams. In mathematical terms, the proportion of female progeny in tier $i$ selected as dams for tier $1\left(q_{i 1}\right)$ must satisfy $\sum_{i=1}^{n} p_{i} q_{i 1}=b p_{1}$ where the standardised phenotypic truncation point in tier $i\left(X_{i 1}\right)$ must satisfy $t_{1}=\mu_{i}+X_{i 1} \sigma_{P i}$ with $\mu_{i}$ and $\sigma_{P i}$ being the mean breeding value and phenotypic standard deviation, respectively, of tier $i$.

\section{Step 3}

Select as dams for tier $j(j=2, \ldots, n)$ all female progeny whose phenotype is not only smaller than the previous common truncation point $t_{j-1}$ but also greater than another common truncation point $t_{j}$ which is chosen such that the best $b P_{j}$ of all female progeny become dams in tiers 1 to $j$ with the best $b P_{j-1}$ in tiers 1 to $j-1$, and where $P_{j}=\sum_{k=1}^{j} p_{k}$. In mathematical terms, determine the proportion of female progeny in tier $i$ selected as dams for tier $j\left(q_{i j}\right)$ using $q_{i j}=Q_{i j}-Q_{i, j-1}$ 
where $Q_{i 1}=q_{i 1}$ and the other proportions $Q_{i j}$ satisfy $\sum_{i=1}^{n} p_{i} Q_{i j}=b P_{j}$ while the standardised phenotype truncation point in tier $i\left(X_{i j}\right)$ must satisfy $t_{j}=\mu_{i}+X_{i j} \sigma_{P i}$ for $i=1, \ldots, n$.

\section{Step 4}

In an analogous manner repeat Steps 2 and 3 to calculate the proportion of male progeny in tier $i$ selected as sires for tier $j\left(q_{i j}^{*}\right)$.

\section{Step 5}

Now calculate the proportion of tier $j$ dams born in tier $i\left(a_{i j}\right)$ and the proportion of tier $j$ sires born in tier $i\left(a_{i j}^{*}\right)$ using the identities

$$
a_{i j}=\frac{q_{i j} p_{i}}{b p_{j}} \quad \text { and } \quad a_{i j}^{*}=\frac{q_{i j}^{*} p_{i}}{a p_{j}}
$$

\section{Step 6}

The mean progeny breeding value of tier $j$ at generation $t+1\left(\mu_{j}^{t+1}\right)$ is

$$
\mu_{j}^{t+1}=\frac{1}{2} \sum_{k}\left[a_{k j}\left(\mu_{k}^{t}+D_{k j}\right)+a_{k j}^{*}\left(\mu_{k}^{t}+D_{k j}^{*}\right)\right]
$$

where $D_{k j}$ and $D_{k j}^{*}$ are the genetic selection differentials of female and male progeny, respectively, who are born in tier $k$ and mated in tier $j$. Now $D_{k j}=i_{k j} h_{k}^{2} \sigma_{P k}$ and $D_{k j}^{*}=i_{k j}^{*} h_{k}^{2} \sigma_{P k}$ where $h_{k}^{2}$ is the heritability in tier $k$ and $i_{k j}\left(i_{k j}^{*}\right)$ is the standardised selection differential for tier $k$ born females (males) who are mated in tier $j$. The standardised selection differentials are calculated assuming progeny phenotype is normally distributed within each tier. Let $S(q)$ denote the standardised selection differential achieved by truncation selection of the best proportion $q$. Then the standardised selection differential of tier $k$ born females mated in tier $j\left(i_{k j}\right)$ is given by $i_{k 1}=S\left(q_{k 1}\right)$ and for $j>1$ by $i_{k j}=\left[Q_{k j} S\left(Q_{k j}\right)-Q_{k, j-1} S\left(Q_{k, j-1}\right)\right] / q_{k j}$, where $Q_{k j}=\sum_{l=1}^{j} q_{k l}$ as in Step 3. The standardised selection differential for males are calculated similarly.

\section{Step 6a}

Finally update all progeny variances taking into account the loss of genetic variance due to selection and the gain in genetic variance due to mixing of groups with different mean breeding values. Proceed as follows. Calculate the mean breeding values of the selected female genetic groups which are born in tier $k$ and either mated in tier $j\left(m_{k j}\right)$ or mated in tiers 1 to $j\left(M_{k j}\right)$ using the equations $m_{k j}=\mu_{k}+i_{k j} h_{k}^{2} \sigma_{P k}$ and $M_{k j}=\mu_{k}+i_{Q_{k j}} h_{k}^{2} \sigma_{P k}$ where $i_{Q_{k j}}=S\left(Q_{k j}\right)$. Now the breeding value variance 
of dams born in tier $k$ which are mated in tier $j\left(V_{A_{k j}}\right)$ is given by $V_{A_{k 1}}=V_{A_{k}} K_{k 1}$ and for $j>1$ by

$$
V_{A_{k j}}=V_{A_{k}}\left(\frac{Q_{k j} K_{k j}-Q_{k, j-1} K_{k, j-1}}{q_{k j}}\right)-\frac{Q_{k, j-1}\left(M_{k, j-1}-m_{k j}\right)^{2}}{Q_{k j}}
$$

where $K_{k j}=1-i_{Q_{k j}}\left(i_{Q_{k j}}-X_{k j}\right) h_{k}^{2}$ and $V_{A_{k}}$ is the variance of progeny breeding values in tier $k$. Now the mean breeding value of all dams used in tier $j$ is $m_{j}=\sum_{k} a_{k j} m_{k j}$. Hence the pooled breeding value variance of all dams used in tier $j\left(V_{F A_{j}}\right)$ is

$$
V_{F A_{j}}=\sum_{k} a_{k j}\left[V_{A_{k j}}+\left(m_{k j}-m_{j}\right)^{2}\right]
$$

where account has been taken of the extra variation due to the differences in mean breeding value of the selected female genetic groups. In a similar manner, the pooled variance of all sires mated in tier $j\left(V_{M A_{j}}\right)$ can be derived. Hence the variance of progeny breeding value in tier $j$ at generation $t+1\left(V_{A_{j}}^{t+1}\right)$ is calculated as follows.

$$
V_{A_{j}}^{t+1}=\frac{1}{4} V_{F A_{j}}^{t}+\frac{1}{4} V_{M A_{j}}^{t}+\frac{1}{2} V_{A}^{0}
$$

where $V_{A}^{0}$ is the initial genetic variance before selection. Using $V_{A_{j}}^{t+1}$ we can calculate the heritability and phenotypic variance in tier $j$ at generation $t+1$.

\section{Step 7}

Now repeat Steps 1 to $6 a$ for the next generation. Stop when the required number of generations is reached.

\section{Random mating - the Bulmer method}

The deterministic prediction of the genetic gain at generation $t+1\left(G_{B}^{t+1}\right)$ for random mating following mass selection is calculated from $G_{B}^{t+1}=\frac{1}{2}[S(a)+$ $S(b)] \sqrt{h_{t}^{2} V_{A}^{t+1}}$ using an obvious notation. Now the additive genetic variance at generation $t+1\left(V_{A}^{t+1}\right)$ is calculated using $V_{A}^{t+1}=\frac{1}{4} V_{F A}^{t}+\frac{1}{4} V_{M A}^{t}+\frac{1}{2} V_{A}^{0}$ where $V_{F A}^{t}$ and $V_{M A}^{t}$ are the additive genetic variance of dams and sires respectively, at generation $t$. To calculate $V_{F A}^{t}$ use the equation $V_{F A}^{t}=V_{A}^{t}\left\{1-S(b)\left[S(b)-X_{F}\right] h_{t}^{2}\right\}$ where $X_{F}$ is the standardised truncation point for females. A similar equation is used for calculating $V_{M A}^{t}$. The heritability at each generation is calculated in the usual manner. 